\title{
Effect of High-frequency Diathermy on Hamstring Tightness
}

\author{
Ye Jin Kim¹, BPT, PT, Joo-Hee Park², PhD, PT, Ji-hyun Kim¹, BPT, PT, Gyeong Ah Moon¹, BPT, PT, \\ Hye-Seon Jeon², PhD, PT
}

${ }^{1}$ Department of Physical Therapy, The Graduate School, Yonsei University, ${ }^{2}$ Department of Physical Therapy, College of Health Science, Yonsei University, Wonju, Korea

\author{
Article Info \\ Received January 14, 2021 \\ Revised January 25, 2021 \\ Accepted January 28, 2021

\section{Corresponding Author} \\ Hye-Seon Jeon \\ E-mail: hyeseonj@yonsei.ac.kr \\ https://orcid.org/0000-0003-3986-2030
}

\section{Key Words}

Diathermy

Hamstring muscles

Muscle tonus

Physical therapy modalities
Background: The hamstring is a muscle that crosses two joints, that is the hip and knee, and its flexibility is an important indicator of physical health in its role in many activities of daily living such as sitting, walking, and running. Limited range of motion (ROM) due to hamstring tightness is strongly related to back pain and malfunction of the hip joint. High-frequency diathermy (HFD) therapy is known to be effective in relaxing the muscle and increasing ROM.

Objects: To investigate the effects of HFD on active knee extension ROM and hamstring tone and stiffness in participants with hamstring tightness.

Methods: Twenty-four participants with hamstring tightness were recruited, and the operational definition of hamstring tightness in this study was active knee extension ROM of below $160^{\circ}$ at $90^{\circ}$ hip flexion in the supine position. HFD was applied to the hamstring for 15 minutes using the WINBACK device. All participants were examined before and after the intervention, and the results were analyzed using a paired t-test. The outcome measures included knee extension ROM, the viscoelastic property of the hamstring, and peak torque for passive knee extension.

Results: The active knee extension ROM significantly increased from $138.8^{\circ} \pm 9.9^{\circ}$ (mean \pm standard deviation) to $143.9^{\circ} \pm 10.4^{\circ}$ after the intervention $(p<0.05)$, while viscoelastic property of the hamstring significantly decreased $(p<0.05)$. Also, the peak torque for knee extension significantly decreased $(p<0.05)$.

Conclusion: Application of HFD for 15 minutes to tight hamstrings immediately improves the active ROM and reduces the tone, stiffness, and elasticity of the muscle. However, further experiments are required to examine the long-term effects of HFD on hamstring tightness including pain reduction, postural improvement around the pelvis and lower extremities, and enhanced functional movement.

\section{INTRODUCTION}

The hamstring functions as both the hip extensor and knee flexor. Because it crosses both the hip and knee, the length of the hamstring affects not only the range of motion (ROM) of hip flexion and knee extension; but also the posture of the pelvis and spine $[1,2]$. Hamstring tightness is a major cause of back injury and dysfunction [3,4]. In particular, forward bending motion is affected by the length of hamstring, and hamstring tightness increases the risk of spinal injury due to mechanical stress [5]. Previous studies have reported that maintaining proper hamstring length prevents excessive flexion of the low back during forward bending, which reduces the risk of injury by reducing anterior shearing forces of the spine [6]. In addition, it can lead to dysfunctions, such as poor posture and abnormal walking stance [7]. According to Wong and Lee [8], because the hamstring is a major dynamic muscle in the pelvic area, tightness of this muscle reduces pelvic anterior tilting, limits hip joint movements, and causes pain. Ultimately, structural and functional changes in the hamstring affect activities of daily living, such as walking and postural control. Therefore, hamstring flexibility is an important contributor to physical health, and plays a role in many activities of daily living such as sitting, walking, and running [9].

Treating muscle tightness is essential for maintaining and improving the posture and preventing injuries during daily life 
activities or exercise [10]. Various stretching techniques, physical agents and medications have been studied as interventions for muscle tightness. Conventionally, manual approaches such as hold-relax, stretching, and soft tissue mobilization have been commonly used to improve hamstring tightness, but the outcomes have been inconsistent. Moreover, manual techniques are physically demanding and can cause musculoskeletal impairments in therapists who apply these techniques. In addition, the most commonly used superficial heat therapy merely heat the skin and subcutaneous tissue to increase blood circulation, but it does not affect the deep tissues. Thus, diathermy therapy is used to relax the muscles by transmitting heat to the muscles [11]. High-frequency diathermy (HFD) therapy, produces heat through molecular vibrations in deep tissues [12]. Previous studies have shown that the application of HFD increase blood circulation and tissue flexibility, resulting in muscle relaxation $[11,13]$. HFD also increases the local tissue temperature to $40^{\circ} \mathrm{C}-45^{\circ} \mathrm{C}$, thus increasing the pain threshold, reducing pain and enabling maximum stretch without damage [14].

Traditional HFD devices, however, have limitations in their application as the treatment electrodes are large and fixed. The WINBACK device (WINBACK 3SE, Villeneuve Loubet, France) is convenient to use as it allows therapists to use their hands and move the electrodes while applying HFD [15]. The device uses the transfer electrode capacitive and resistive (TECAR) technique, which operates within the long-wave radio frequency range at $0.5 \mathrm{MHz}$, and can apply both capacitive energy transfer (CET) with shallow penetration and resistive energy transfer (RET) with deep penetration depths. CET is effective in the treatment of soft tissue injuries because it uses coated electrodes to transmit heat quickly to the skin and superficial muscles, while RET uses uncoated electrodes, so it can gradually transmit heat to deep tissues, making it effective in the treatment of hard tissues such as tendons and joints.

Previous studies have shown that WINBACK increased the

Table 1. General characteristics of the participants $(N=24)$

\begin{tabular}{lc}
\hline \multicolumn{1}{c}{ Variables } & Data \\
\hline Age $(\mathrm{y})$ & $25.9 \pm 2.3$ \\
Height $(\mathrm{cm})$ & $170.3 \pm 9.5$ \\
Weight $(\mathrm{kg})$ & $73.9 \pm 14.4$ \\
Body mass index $\left(\mathrm{kg} / \mathrm{m}^{2}\right)$ & $25.3 \pm 3.8$ \\
Active knee extension range of motion $\left({ }^{\circ}\right)$ & $138.8 \pm 9.9$ \\
\hline
\end{tabular}

Values are presented as mean \pm standard deviation. length of the shortened gastrocnemius (GCM) and improved back pain [16,17]. However, no previous studies have been conducted on the relaxation effect of HFD on hamstring tightness. Therefore, the purpose of this study was to investigate the effects of HFD on the active knee extension ROM and hamstring tone and stiffness in participants with hamstring tightness.

\section{MATERIALS AND METHODS}

\section{Participants}

Twenty-four participants with hamstring tightness less than $160^{\circ}$ in the active knee extension test volunteered for this study. The mean age was $25.9 \pm 2.3$ years. The exclusion criteria were (1) neuromotor or musculoskeletal impairments, (2) history of surgery in the lower extremities, (3) decreased temperature and pain sense and a metal insert in the HFD therapy area, and (4) difficulty in expressing thermal sense. All participants read a written explanation about the study and signed an informed consent form approved by Yonsei University Mirae Campus Institutional Review Board (approval no. 1041849202008-BM-095-02). The demographic data of the participants are presented in Table 1.

\section{Intervention}

In this study, HFD was applied to the hamstring using the TECAR technique with the WINBACK device (Figure 1). In particular, the TECAR technique involves attaching a fixed

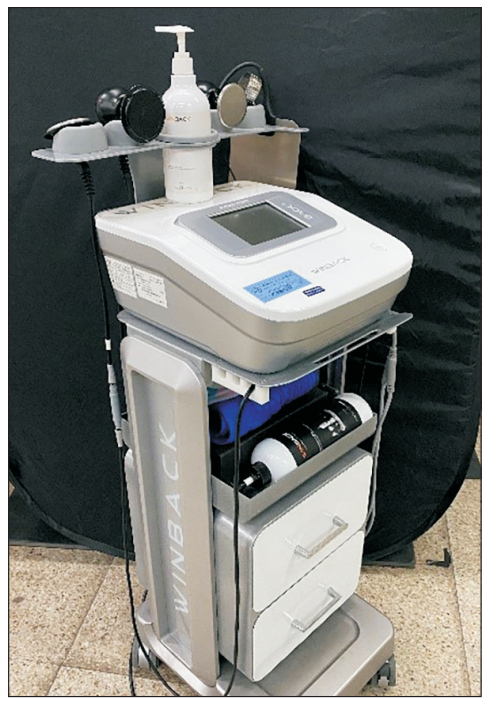

Figure 1. WINBACK 3SE device. 
electrode to the participant's body and applying a mobile electrode to the treatment area it allows therapists' to simultaneously use the device and their hands. To apply TECAR therapy to the hamstring muscles, the fixed electrode was applied to the quadriceps muscle, and the active electrode was applied to the hamstring with the participant in the prone position (Figure 2). The intervention was conducted for 15 minutes; the RET mode was applied for 10 minutes after 5 minutes of CET mode application. The current intensity was set to a level that the participants found comfortable and warm.

\section{Outcome Measures}

The Active knee extension ROM, peak torque for passive knee extension, and muscle tone related variables were measured before and after 15 minutes of TECAR therapy.

\section{1) Active knee extension ROM test}

The active knee extension ROM test was conducted to measure the hamstring length before and after the intervention, and the knee joint extension angle was measured using a universal goniometer. The test started with a $90^{\circ}$ flexion of the hip and knee joints, with the ankle in the neutral position and the participant in the supine position. The cross-bar was used to maintain $90^{\circ}$ flexion of the hip joint, and the angle was measured with the knee in full extension. During the test, the pelvis and opposite thigh were secured with straps to maintain lordotic curve of lumbar and prevent compensatory movement (Figure 3). The stable arm of the goniometer was positioned laterally near the epicondyle of the femur, and the moving arm was positioned over the line drawn from the fibular head to the lateral malleolus.

\section{2) Peak torque for passive knee extension}

The Biodex System Isokinetic Dynamometer (Biodex Medical, Shirley, NY, USA) was used to assess the peak torque for passive knee extension. The participant sat on the Biodex dynamometer chair, and their trunk, pelvis, and thigh were secured with straps to minimize compensation movement. After aligning the dynamometer axis to the participant's knee joint axis, the calf was fixed with straps (Figure 4). The dynamometer was set to repeat five times at an angular velocity of $5 \%$ in passive mode, and the average value was obtained by measuring it three times. The knee extension angle was set to the full ROM of the participants.

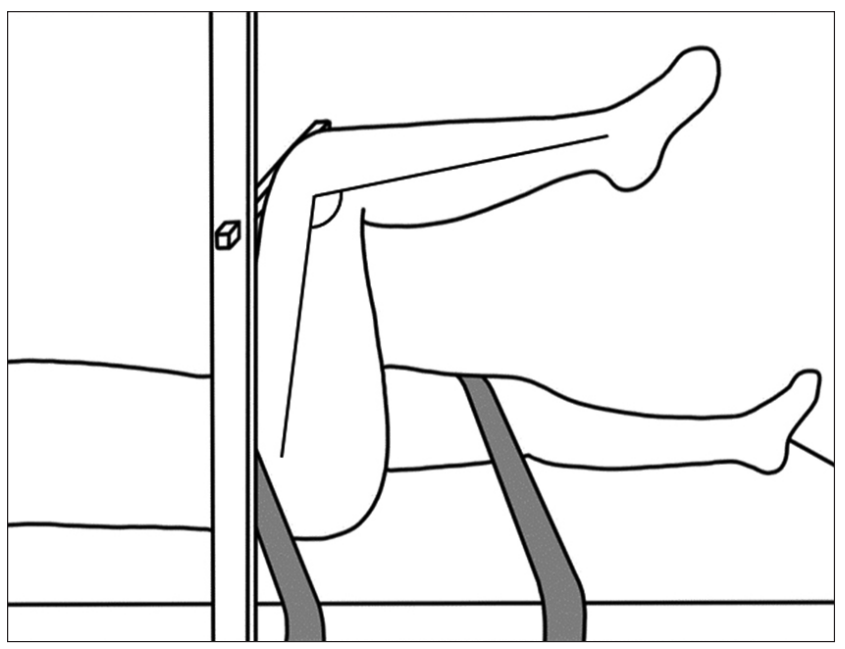

Figure 3. Active knee extension ROM test. ROM, range of motion.

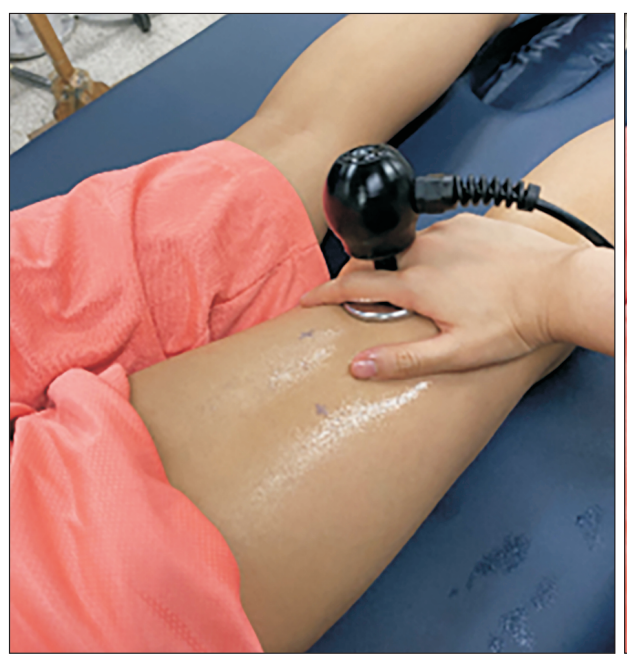

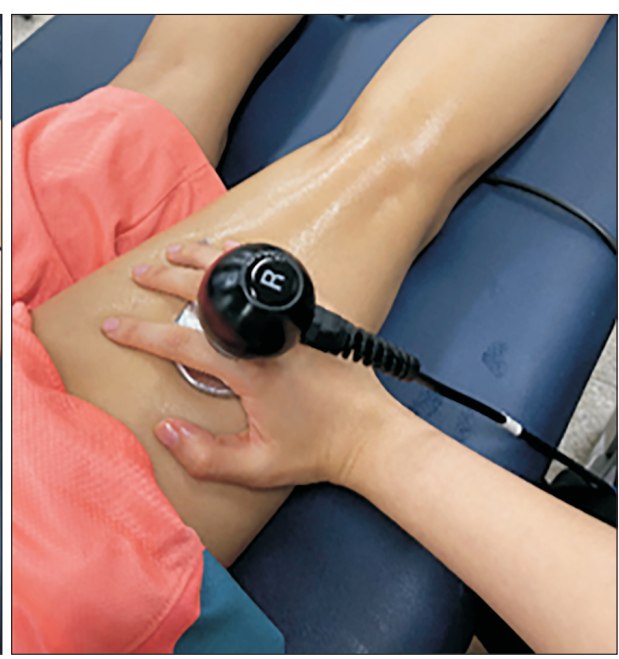

Figure 2. TECAR therapy to the hamstring. TECAR, transfer electrode capacitive and resistive. 


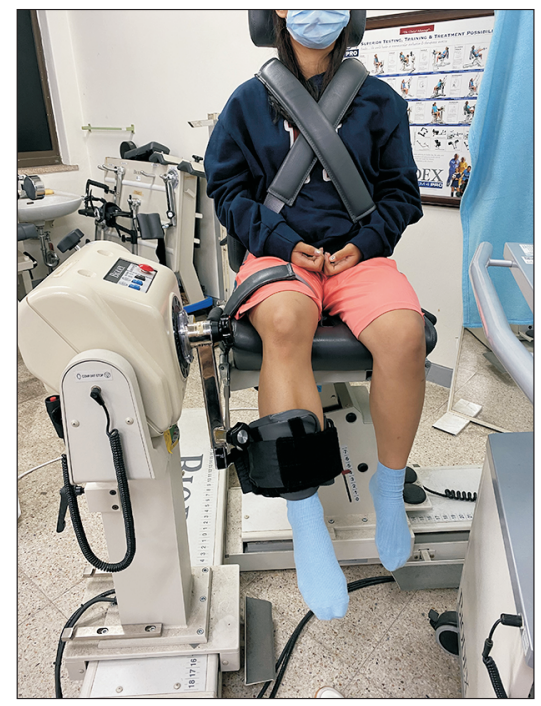

Figure 4. Biodex system isokinetic dynamometer.

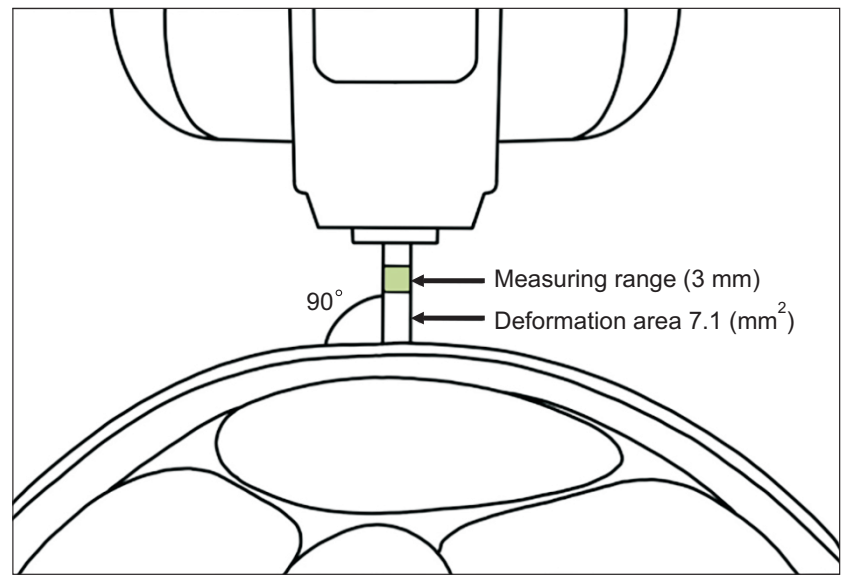

Figure 5. Description of the MyotonPRO structure.

\section{3) Viscoelastic property of the hamstring}

MyotonPRO (Myoton AS, Tallinn, Estonia) was used to measure the viscoelastic properties of the muscle (Figure 5). The MyotonPRO is a device that can measure muscle tone(F), dynamic stiffness(S), elasticity(D), relaxation time(R), and creep(C); it has high reliability in evaluating muscle characteristics [18]. At the beginning of the assessment, the participants rested in the prone position without voluntary contraction of the muscles. The data were collected from the semitendinosus and biceps femoris muscles, and each muscle belly was marked using a pen so that the same point could be measured. The device was placed perpendicular to the skin surface at the marked point and pressed until the measuring range $(3 \mathrm{~mm})$ marked on the probe was reached. The pre-compression at this time was $0.18 \mathrm{~N}$ and the device automatically made five
Table 2. Active knee extension ROM

\begin{tabular}{cccc}
\hline Variable & Pre-test & Post-test & p-value \\
\hline ROM $\left(^{\circ}\right)$ & $138.8 \pm 9.9$ & $143.9 \pm 10.4$ & 0.00 \\
\hline
\end{tabular}

Values are presented as mean \pm standard deviation. ROM, range of motion.

Table 3. Peak torque for passive knee extension

\begin{tabular}{cccc}
\hline Variable & Pre-test & Post-test & p-value \\
\hline Peak torque $(\mathrm{N} / \mathrm{m})$ & $20.3 \pm 4.0$ & $19.3 \pm 3.5$ & 0.03 \\
\hline Values are presented as mean \pm standard deviation.
\end{tabular}

Values are presented as mean \pm standard deviation.

taps when placed within the measuring range. The impulse force during tapping was $0.4 \mathrm{~N}$, and impulse time was $15 \mathrm{~ms}$. In this study, three variables (muscle tone, stiffness, and elasticity) were measured and analyzed. According to the manufacturer's manual, muscle tone refers to the oscillation frequency of a muscle in its resting state without any voluntary contraction, and dynamic stiffness refers to the biomechanical characteristic of a muscle that allows it to resist external forces that deform its initial shape, and elasticity and the ability of the muscle to recover its initial shape after removal of the external force [19].

\section{Statistical Analysis}

The collected data were statistically analyzed using Windows SPSS version 24.0 (IBM Co., Armonk, NY, USA). A paired t-test was used to compare the means of the dependent variables before and after the intervention; the significance level was set to 0.05 .

\section{RESULTS}

\section{Active Knee Extension ROM}

All participants demonstrated significant improvement in the active knee extension ROM after the application of HFD ( $p<$ 0.05) (Table 2).

\section{Peak Torque for Passive Knee Extension}

The peak torque decreased significantly after the application of HFD $(\mathrm{p}<0.05)$ (Table 3$)$.

\section{Viscoelastic Property Variables of the Hamstring}

The viscoelastic property variables of muscle tone, stiffness, and elasticity obtained from the MyotonPRO had significantly 
decreased $(\mathrm{p}<0.05)$ (Table 4, Figure 6).

\section{DISCUSSION}

The present study examined the clinical effects of applying HFD using the WINBACK device, on participants with hamstring tightness. With 15 minutes of HFD application to the hamstring, the active knee extension ROM increased, and the peak torque for passive knee extension, muscle tone, stiffness, and elasticity decreased. The Decrease in muscle tone, stiffness, elasticity, and peak torque indicates the release of hamstring muscle tightness, resulting in an increase in knee extension ROM.

Hamstring tightness is known to result in reduced range of motion and increased viscoelasticity and contractility of soft tissues [20]. The decrease in peak torque in this study indicates that after HFD application, subjects were able to move from specific torque level to a wider range of motion, and this change in torque can be attributed to increased local temperature and changes in muscle viscoelasticity due to HFD, resulting in muscle relaxation [21,22]. The decrease in viscoelasticity property variables measured by the MyotonPRO also seems to be due to tissue changes in the hamstring caused by HFD. In this study, muscle tone, stiffness, and elasticity were reduced, which was consistent with previous study of HFD in GCM [16]. Yeatman et al. [23] reported that temperature and muscle elasticity were inversely proportional to each other, and the decrease in muscle elasticity in the study was attributed to increased deep heat due to HFD.

It is known that high-frequency current passing through human tissue is converted to thermal energy, resulting in deeptissue heat and pain reduction, microcirculation, and vasodilatation. HFD is beneficial for the release of tight muscles, ligaments, and tendons [24]. Fifteen minutes of HFD application increased the local temperature of the body by $3^{\circ} \mathrm{C}-5^{\circ} \mathrm{C}$, and the increase in temperature was maintained for 3-4 hours after treatment [25]. Lehmann stated that optimal functional recovery of the tissue and maximum stretching can be achieved without tissue damage when the local temperature of the connective tissue is $40^{\circ} \mathrm{C}-50^{\circ} \mathrm{C}$ [26]. Therefore, HFD is an effective intervention to maximize the effectiveness of muscle stretching. Therefore, future studies propose to confirm the effectiveness of applying HFD and muscle stretching simultaneously.

Because this study only investigated the short-term effects of HFD without a control group, experiments on the long-term effectiveness of HFD with a control group must be considered

Table 4. Viscoelastic property of hamstrings

\begin{tabular}{|c|c|c|c|c|}
\hline Muscle & Variables & Pre-test & Post-test & p-value \\
\hline \multirow[t]{3}{*}{ Biceps femoris } & Tone $(\mathrm{Hz})$ & $14.4 \pm 1.0$ & $13.9 \pm 1.0$ & 0.00 \\
\hline & Stiffness (N/m) & $244.8 \pm 28.6$ & $230.6 \pm 29.2$ & 0.00 \\
\hline & Elasticity & $1.1 \pm 0.1$ & $1.0 \pm 0.1$ & 0.00 \\
\hline \multirow[t]{3}{*}{ Semitendinosus } & Tone $(\mathrm{Hz})$ & $14.7 \pm 1.4$ & $14.0 \pm 1.4$ & 0.00 \\
\hline & Stiffness $(\mathrm{N} / \mathrm{m})$ & $247.4 \pm 34.2$ & $231.7 \pm 34.3$ & 0.00 \\
\hline & Elasticity & $1.1 \pm 0.1$ & $1.0 \pm 0.1$ & 0.00 \\
\hline
\end{tabular}

Values are presented as mean \pm standard deviation.
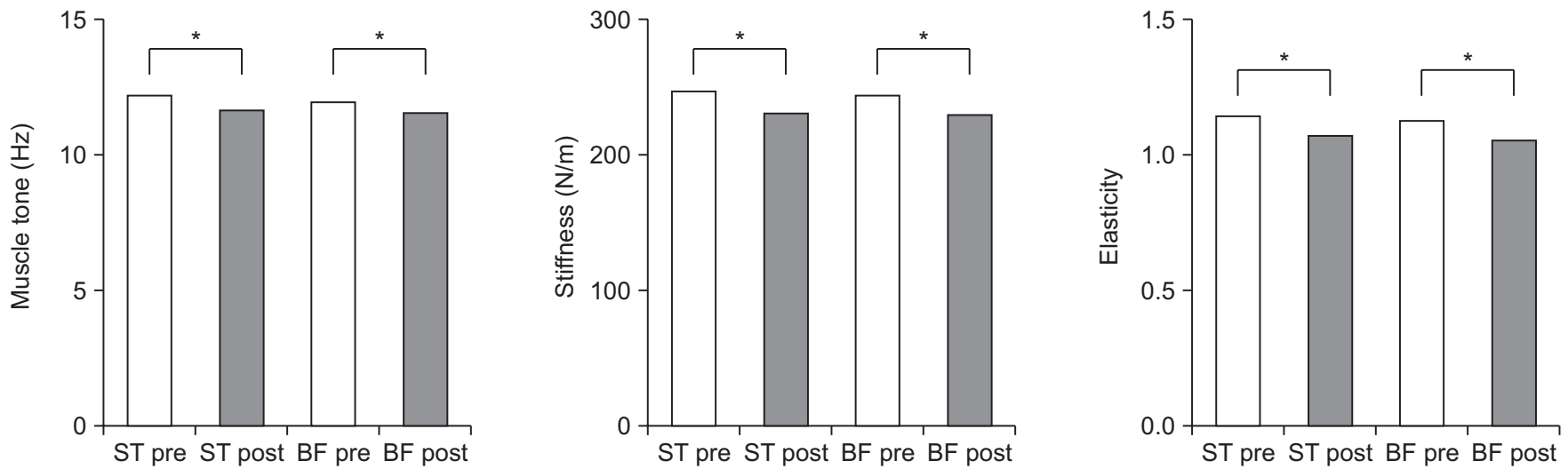

Figure 6. Viscoelastic property of hamstring. ST: semitendinosus; BF: biceps femoris; ${ }^{*} p<0.05$. 
in future studies. Furthermore, future studies need to include outcome measures to examine the dynamic functional changes after HFD application to the hamstring, such as jumping and running.

\section{CONCLUSIONS}

Our findings provide evidence that applying HFD for 15 minutes to tight hamstrings immediately improves the active knee extension ROM and reduces the peak torque, tone, stiffness, and elasticity of the hamstring. However, further experiments are required to examine the long-term effects of HFD on hamstring tightness, including possible pain reduction, postural improvement around the pelvis and lower extremities, and enhanced functional movement.

\section{ACKNOWLEDGEMENTS}

This study was supported by the "Brain Korea 21 FOUR Project", the Korean Research Foundation for Department of Physical Therapy in the Graduate School of Yonsei University.

\section{CONFLICTS OF INTEREST}

No potential conflict of interest relevant to this article was reported.

\section{AUTHOR CONTRIBUTIONS}

Conceptualization: YJK, JHP, HSJ. Data curation: YJK, GAM, JK, JHP. Formal analysis: YJK, GAM, JK, JHP, HSJ. Investigation: YJK, GAM, JK, JHP. Methodology: YJK, GAM, JK, JHP, HSJ. Project administration: YJK, JHP, HSJ. Resources: YJK, GAM, JK, JHP. Supervision: YJK, GAM, JK, JHP, HSJ. Validation: YJK, HSJ. Visualization: YJK. Writing - original draft: YJK, HSJ. Writing - review \& editing: YJK, HSJ.

\section{ORCID}

Ye Jin Kim, https://orcid.org/0000-0003-0802-6195

Joo-Hee Park, https://orcid.org/0000-0001-9545-1362

Ji-hyun Kim, https://orcid.org/0000-0003-2701-4317

Gyeong Ah Moon, https://orcid.org/0000-0001-7906-811X

\section{REFERENCES}

1. Congdon R, Bohannon R, Tiberio D. Intrinsic and imposed hamstring length influence posterior pelvic rotation during hip flexion. Clin Biomech (Bristol, Avon) 2005;20(9):947-51.

2. López-Miñarro PA, Muyor JM, Belmonte F, Alacid F. Acute effects of hamstring stretching on sagittal spinal curvatures and pelvic tilt. J Hum Kinet 2012;31:69-78.

3. Gajdosik RL, Hatcher CK, Whitsell S. Influence of short hamstring muscles on the pelvis and lumbar spine in standing and during the toe-touch test. Clin Biomech (Bristol, Avon) 1992;7(1):38-42.

4. Wang SS, Whitney SL, Burdett RG, Janosky JE. Lower extremity muscular flexibility in long distance runners. J Orthop Sports Phys Ther 1993;17(2):102-7.

5. Esola MA, McClure PW, Fitzgerald GK, Siegler S. Analysis of lumbar spine and hip motion during forward bending in subjects with and without a history of low back pain. Spine (Phila Pa 1976) 1996;21(1):71-8.

6. Johnson EN, Thomas JS. Effect of hamstring flexibility on hip and lumbar spine joint excursions during forward-reaching tasks in participants with and without low back pain. Arch Phys Med Rehabil 2010;91(7):1140-2.

7. Kisner C, Colby LA, Borstad J. Therapeutic exercise: foundations and techniques. Philadelphia: F.A. Davis; 2018.

8. Wong TK, Lee RY. Effects of low back pain on the relationship between the movements of the lumbar spine and hip. Hum Mov Sci 2004;23(1):21-34.

9. Stephens J, Davidson J, Derosa J, Kriz M, Saltzman N. Lengthening the hamstring muscles without stretching using "awareness through movement". Phys Ther 2006;86(12):1641-50.

10. Ogura Y, Miyahara Y, Naito H, Katamoto S, Aoki J. Duration of static stretching influences muscle force production in hamstring muscles. J Strength Cond Res 2007;21(3):788-92.

11. Chung SG. Rehabilitative treatments of chronic low back pain. J Korean Med Assoc 2007;50(6):494-506.

12. Lee M, Oh JS. Effect of women's abdominal obesity management induced by frequency figure of high frequency instrument. Asian J Beauty Cosmetol 2008;6(1):43-58.

13. Hawamdeh M. The effectiveness of Capacitive Resistive Diathermy (Tecartherapy ${ }^{\circledR)}$ in acute and chronic musculoskeletal lesions and pathologies. Eur J Sci Res 2014;118(3):33640.

14. Lehmann JF, Warren CG, Scham SM. Therapeutic heat and 
cold. Clin Orthop Relat Res 1974;(99):207-45.

15. Kwon K, Kim CM, Park SJ. The effect of diathermy treatment through physiotherapist's hand on the pain and disability index in chronic lumbar pain patients. J Korea Converg Soc 2018;9(2):289-94.

16. Kim J, Park J, Yoon H, Lee J, Jeon H. Immediate effects of highfrequency diathermy on muscle architecture and flexibility in subjects with gastrocnemius tightness. Phys Ther Korea 2020;27(2):133-9.

17. Ribeiro S, Henriques B, Cardoso R. The effectiveness of Tecar therapy in musculoskeletal disorders. Int J Public Health Health Syst 2018;3(5):77-83.

18. Pruyn EC, Watsford ML, Murphy AJ. Validity and reliability of three methods of stiffness assessment. J Sport Health Sci 2016;5(4):476-83.

19. Myoton Ltd. MyotonPRO user manual. Tallinn: Myoton AS; 2012.

20. Ha SH, Lee GC. Potential benefits of a selective region highfrequency diathermy with therapeutic exercises on older persons with degenerative knee osteoarthritis. Res Sq. [Preprint]. 2020 [cited 2020 Oct]. Available from: https://doi. org/10.21203/rs.3.rs-117670/v1.

21. Kitchen SS, Partridge CJ. Infra-red therapy. Physiotherapy 1991;77(4):249-54.

22. Reid DA, McNair PJ. Effects of an acute hamstring stretch in people with and without osteoarthritis of the knee. Physiotherapy 2010;96(1):14-21.

23. Yeatman LA Jr, Parmley WW, Sonnenblick EH. Effects of temperature on series elasticity and contractile element motion in heart muscle. Am J Physiol 1969;217(4):1030-4.

24. Ganzit GP, Stefanini L, Stesina G. Tecar therapy in the treatment of acute and chronic pathologies in sports. BTL [Internet]. Boston: 2000 [cited 2020 Oct]. Available from: https:// www.tr-therapy.com/scientific-support-tecar-therapy-in-thetreatment-of-acute-and-chronic-pathologies-in-sports.

25. Gutmann AZ. Recientes avances en fisioterapia. Barcelona: JIMS; 1993;321-32.

26. Lehmann JF, De Lateur BJ. Diathermy and superficial heat, laser and cold therapy. In: Krusen FH, Kottke FJ, Lehmann JF, editors. Krusen's handbook of physical medicine and rehabilitation. 4th ed. Philadelphia: Saunders; 1990;283-435. 